\title{
When Efficiency Matters: Towards Quality of Context-aware Peers for Adaptive Communication in VANETs
}

\author{
Ansar-Ul-Haque Yasar, Koosha Paridel, Davy Preuveneers and Yolande Berbers
}

\begin{abstract}
In the near future vehicles will be equipped with embedded communication capabilities in order to perform context-sensitive tasks such as traffic flow control and incident avoidance. In large scale vehicular networks it is of vital importance that the information exchanged between the vehicles meets a certain level of quality so that they can make informed automated decisions. In this paper we define Quality of Context (QoC) and Peer Reputation (PR) for nodes in vehicular networks and propose ways to apply them for efficient communication. Thus, we provide a two-fold solution in which on the one hand we focus on the quality of the information and on the other hand we aim at determining the reputation of the nodes involved in the communication. This helps to eliminate the use of erroneous, ambiguous and imprecise information provided by unknown entities. Our simulated experiments show that our mechanism significantly reduces network traffic usage, eases out the decision making process and guarantees a minimum level of quality.
\end{abstract}

\section{INTRODUCTION}

As a result of rapid advancements in embedded technology, vehicles are being equipped with wireless communication capabilities. Vehicles will be able to communicate with each other in order to perform context-sensitive tasks. Application areas include accident avoidance, incident notification, congestion monitoring and parking space allocation [4].

In large scale, highly dynamic and mobile ad hoc networks such as vehicular networks it is crucial that the nodes receive the right information at the right time and the right place to make informed decisions. However, due to the ephemeral nature of the vehicular networks we face several challenges such as; (i) unknown source of information, (ii) no guarantee on the quality of information, (iii) erroneous information, (iv) ambiguous information, (v) imprecise information, (vi) high network traffic, (vii) intensive decision making process.

In this paper we define Quality of Context (QoC) and Peer Reputation (PR) for nodes in vehicular networks and propose ways to apply them for efficient communication. Thus, we provide a two-fold solution in which on the one hand we focus on the quality of the information and on the other hand we aim at determining the reputation of the nodes involved in the communication. We propose methods to calculate aggregated quality after data fusion along with ways to apply QoC and PR for communication in VANETs. On the one hand, QoC is the weighted average of all the quality constraints related to the context information. The

A. Yasar, K. Paridel, D. Preuveneers and Y. Berbers are with Department of Computer Science, Katholieke Universiteit Leuven, Celestijnenlaan 200A, B-3001 Heverlee, Belgium. \{ansarulhaque.yasar, koosha.paridel, davy.preuveneers, yolande.berbers\}@cs. kuleuven.be value of the weights varies and depends on the application area in question. On the other hand, PR is the trustworthiness of a node involved in the communication. Our mechanism makes the following contributions as shown in Figure 1 and discussed below:

- Define bounds of acceptable information: We define the bounds of acceptable quality and ways to measure QoC.

- Filter-out more messages, reducing network traffic: We filter-out messages with low QoC to reduce network traffic which allows us to utilize our communication resources for propagating messages with better quality.

- Ease the decision making process: By filtering messages with a QoC below our minimum threshold, we reduce the number of messages that a node receives. Thus, the decision making process is simplified in nodes.

- Guarantee a minimum quality of information: Computing the QoC for messages, setting a minimum threshold for the QoC that each message should have, and filtering messages which do not satisfy this limit guarantees a minimum quality of information.

The rest of the paper is organized as follows: In section II, we present our motivating scenario. In section III, we present the requirements for QoC and PR in a large scale vehicular network. We also describe our scheme to evaluate the quality of context-aware peers for efficient communication in VANETs. Simulated experiments and results are presented in section IV. In section V, we discuss related work in comparison to our proposed scheme. Last but not the least we present our conclusion with suggestions for future work in section VI.

\section{Motivating Scenario}

Bob is a journalist working and living in Leuven. For his profession he has to travel a lot and most of the cases he uses his car because it gives him the flexibility he needs. His car is equipped with an on-board computing platform with communication and navigation capabilities. This onboard computing unit maintains a driving-profile for each

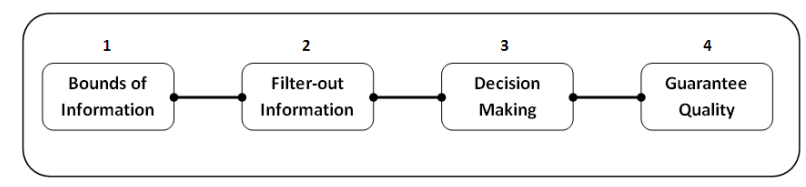

Fig. 1. The figure depicts the sequential steps to ensure the quality of information. 


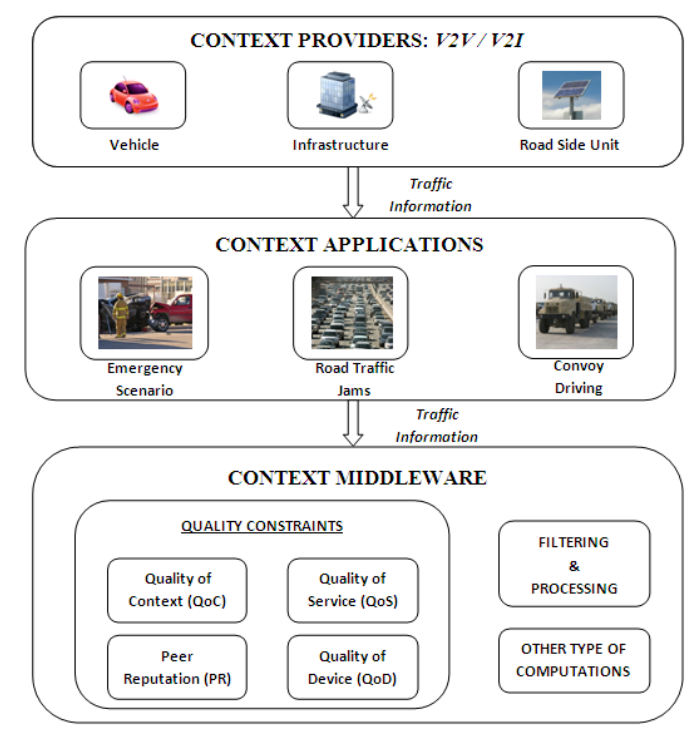

Fig. 2. Quality computation at the middleware layer in a large scale vehicular network.

driver based on their everyday driving activities. The drivingprofile contains the information like the user-profile of the driver, GPS traces of the everyday driving activity and other hardware specifications e.g. model of the car being driven. In large scale vehicular networks, we consider nodes with similar driving-profiles as friends. The higher percentage of the profile match shows a stronger friendship relation between nodes in VANETs.

Today Bob has a meeting in Brussels. He leaves at $8 \mathrm{am}$ and follows the GPS directions. This time in the morning is usually a busy period on the highway. While on the way to Brussels he finds that Alice's car is in the opposite direction and is going towards Leuven. From their drivingprofiles, Alice's car detected that some cars including Bob's car are interested in going towards Brussels. Alice's car has information about a traffic accident on the highway towards Brussels that caused a traffic congestion and it sends this information to all the interested cars in the vicinity. Bob's car receives this information and the on-board computing platform present in the car immediately recalculates the route to the destination based on the overall quality of the information and reputation of the provider.

As Bob is approaching his destination the system checks for available parking spots. It receives two messages (1) from a friend who just left the same parking lot saying that there is a vacant spot, and (2) another from an unknown nearby car saying that there is no free space available. The system again calculates the overall quality of both messages received and accepts the information provided by the friend's car based on a higher degree of quality. Later the system sends a reservation request to the parking meter and routes the car towards the reserved parking spot. It also provides a positive feedback to the car which provided information above the minimum quality threshold and a negative feedback to the other car.
There are different types of interactions in this scenario either in the form of a query or an informative message which are listed as under:

1) What is the quality of the information received?

2) What is the reputation of the information provider?

3) In case of multiple information providers, which one to select?

Our approach provides solutions to these questions. The details are explained later in the paper.

\section{Quality of CONTEXT-AWARE PEers FOR EFFICIENT COMMUNICATION IN VANETS}

In this paper, we define Quality of Context to be used by peers (i.e. vehicles) in context applications for efficient communication as illustrated in Figure 2. Communication between vehicles in VANETs deals with several issues. Addressing these issues helps the communication system to become more efficient and assists the vehicles to access finegrained information while using less effort. These issues are:

- Unknown source of information: When communicating in vehicular networks, most of the received messages come from vehicles which we do not know and we are not aware of their existence. This can cause problems. Some vehicles can produce incorrect or imprecise information for some reasons such as incorrect sensor readings. Without keeping track of the vehicles, as long as they are our direct neighbors, we cannot predict what kind of information we are going to get from them.

- Erroneous information: Context information in a message such as time and location of creation can be incorrect. We can not always recognize whether a message is erroneous or not, but when we do, we should make precautions when dealing with other messages from the same source.

- Ambiguous information: Lacking enough detail can make context information to be vague and to have more than one interpretation. For example, information about a traffic jam in a highway without specifying in which direction of the highway is ambiguous and can be interpreted as a relevant or irrelevant message. Being ambiguous reduces the quality of the context information in a message.

- Imprecise information: Lacking accuracy and precision also reduces the quality of the context information. For example, information about an accident on a road without specifying the exact location complicates the decision making process.

Due to the issues discussed earlier, the messages that a vehicle receives can be of any quality. A message can be totally incorrect or highly imprecise, which may result into lowering the quality of information. Moreover, the high number with unknown quality of the messages can cause:

- High network traffic utilization: Communication between vehicles in a large scale vehicular network can cause a lot of network traffic. High network traffic 


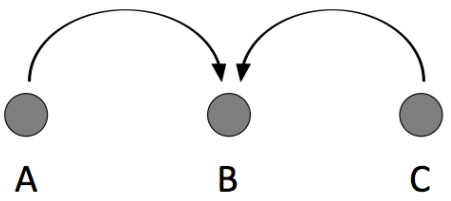

\begin{tabular}{|c|c|c|c|c|c|}
\hline Message & PR & QoC & Aggregated Quality & New PR & Accept \\
\hline A $>$ B & 0.40 & 0.80 & 0.60 & 0.56 & Yes \\
\hline C $>$ B & 0.60 & 0.90 & 0.75 & 0.90 & Yes \\
\hline A $>$ B & 0.56 & 0.90 & 0.73 & 0.84 & No \\
\hline
\end{tabular}

Fig. 3. An example of using aggregated quality. Node A and Node C are sending messages to Node B. Aggregated quality is calculated based on QoC and PR. Node B keeps the message with the highest aggregated quality and discards the rest, even though they have an equal or higher QoC.

causes problems in timely message delivery due to increased transmission delays. A considerable portion of these messages in the network may become irrelevant due to late arrival.

- Intensive decision making process: Each received message in a vehicle needs to be processed before deciding whether it should be used, forwarded or dropped. Processing messages is time and resource intensive. Thus, processing a massive amount of messages is not desirable and we prefer to drop messages with lower quality as soon as possible rather than propagating them in the network.

In our system we define Quality of Context and Peer Reputation. Therefore, we provide a two-fold solution which the former quality reflects upon the latter. On the one hand, Quality of Context describes a measure for relevance of information that a message carries. There are several factors which effect the Quality of Context, such as timeliness and completeness of information. On the other hand, Peer Reputation keeps a history of nodes by tracking the Quality of Context of the messages which originated from these nodes. A message with a low Quality of Context decreases the Peer Reputation for its sender and a message with a high Quality of Context increases it. Moreover, we define a third factor called Aggregated Quality which is calculated based on the Quality of Context and the Peer Reputation. Introducing Aggregated Quality helps us to further optimize our scheme. Each time that a message is delivered at a node, the node computes Aggregated Quality of that message and keeps this information. Later on, upon receiving a message with the same topic of interest, we compute Aggregated Quality for the new message and if it is less than what we have already seen we drop the message. This way, we prevent lower quality messages with the same topic to be propagated in the network while we already have a higher quality information for a specific topic. Figure 3 shows an example of dropping message based on Aggregated Quality.

\section{A. Factors involved in determining Quality of Context $(Q o C)$}

Quality of Context is the weighted average of all the quality constraints related to the contextual information. The value of the weights is variable and depends on the

\begin{tabular}{|l|l|l|l|l|l|l|l|}
\hline ID & Src & Dst & TimeStamp & Type & HopCount & Controllnfo & Payload \\
\hline
\end{tabular}

(a) Message structure

\begin{tabular}{|l|l|l|l|l|l|}
\hline Time & Location & Topic & Priority & TTL & Data \\
\hline
\end{tabular}

(b) Payload structure

Fig. 4. Upper figure depicts the structure of a message which is exchanged between nodes (vehicles) in our system. The lower figure shows the contents of the payload for a traffic flow control application. The payload structure varies according to the application demand.

application area in question. The initial weight values are assigned by the system analysts and are identical for all messages. We define a mechanism to quantify the quality of the messages being disseminated in VANETs and to eliminate the ones with QoC lower than the acceptable threshold. This threshold is static and is set based on the requirements of the application. In order to compute the QoC, we need to examine the message or the information which is being disseminated in a network. There are several factors involved in determining the QoC which are mentioned as under.

- Temporal Relevance(TR): This quality requirement indicates the timeliness of the contextual information. This involves both quick response times as well as having up-to-date information. It is quite critical that the information reaches the destination on-time and every temporally irrelevant information should be discarded by the system. In equation 2, TTL is the total lifespan of a message $m$. The lifespan of a message is set by the sending node which is expressed in seconds. A message becomes irrelevant after its lifespan expires.

$$
\begin{gathered}
\text { Freshness }(m)=\text { ReceivedTime }(m)-\operatorname{SentTime}(m) \\
T R(m)=\left\{\begin{aligned}
1-(\text { Freshness }(m) / T T L(m)) \\
\quad \text { if }(\text { Freshness }<T T L) \\
0, \quad \text { if }(\text { Freshness }>\text { TTL })
\end{aligned}\right.
\end{gathered}
$$

For example, a message which is sent from a car at $8: 00 \mathrm{pm}$ about a traffic incident having a life span of 600 seconds, is received at 8:02pm. This message has a freshness of 120 seconds and therefore the Temporal Relevance of the message is 0.80 .

- Completeness of Information(C): The contextual information which is disseminated in a network is composed of a number of attributes based on the application. Each attribute has a distinct weight $\omega$ based on the requirements of the application. Figure 4(b) illustrates these attributes for a traffic flow control application. For example, in the case of a traffic jam, the exact location is more important than the exact time. Thus, the location has a higher weight as compared to the time (e.g. $\omega_{\text {location }}=2, \omega_{\text {time }}=1$ ).

$$
C(m)=\frac{\Sigma_{n}\left(\omega_{n} \times \operatorname{Attrib}(m)\right)}{\Sigma_{n}\left(\omega_{n}\right)}
$$

- Priority of Information(P): This quality requirement implies the importance or the priority of a contextual 
information in a specific scenario. For example, information provided by the infrastructure and information for real-time applications has priority over other types of nodes in a network.

$$
P(m)_{R E L A T I V E}=\left(P(m)_{A B S} / P_{M A X}\right)
$$

- Spatial Relevance(SR): This quality requirement indicates the distance and direction of the source and the destination nodes. Correctness of the contextual information of a peer is affected by the spatial relevance. Contextual information may be more relevant if received from a neighboring node moving in the same direction as compared to a faraway node or a node moving in the opposite direction.

$$
S R(m)=\left\{\begin{array}{cc}
1-\left(\operatorname{dist}(\text { Src }, \text { Dst }) / \text { dist }_{\text {Max }}\right), \\
& \text { if }(\text { OccurTime }<\text { ObsTime }) \\
0, & \text { if }(\text { OccurTime }>\text { ObsTime })
\end{array}\right.
$$

where,

$$
\operatorname{dist}(s, d)=\sqrt{(x(s)-x(d))^{2}+(y(s)-y(d))^{2}}
$$

- Quality of Context(QoC): QoC is the weighted average of all the quality constraints related to the contextual information. We can assign variable weights $\omega$ for each of the constraints to specify their importance.

$$
\begin{aligned}
& Q o C(m)= \\
& \frac{\left.\omega_{T R} T R(m)+\omega_{C} C(m)+\omega_{P} P(m)+\omega_{S R} S R(m)\right)}{\omega_{T R}+\omega_{C}+\omega_{P}+\omega_{S R}}
\end{aligned}
$$

For example, an ambulance sends a message to the other vehicles ahead of it asking them to clear-out the road. This message has more priority as compared to the messages sent from the other vehicles. In order to increase the effectiveness of the messages sent from an ambulance we give higher weight to the priority parameter $\left(\omega_{P}\right)$. Therefore, the weights will be: $\omega_{T R}=$ $1, \omega_{C}=1, \omega_{P}=2, \omega_{S R}=1$.

\section{B. Factors involved in determining Peer Reputation $(P R)$}

In order to compute PR, we need to evaluate the trustworthiness of a node based on the participation with other nodes. We are dealing with large scale vehicular networks which are ephemeral in nature so we cannot depend on long historical data. Therefore, in our system each node has a default PR value which is awarded or penalized based on the quality of the information provided to other peers.

- Peer Reputation(PR): In order to be able to determine the correctness of information, it is crucial to take into account the reputation of the nodes involved in communication. This quality requirement indicates the value of trust a node has based on the historical interactions. Each node in a network has a certain basic reputation value which is either increased or decreased based on the quality of the information provided. Upon receiving a message from a node its reputation value is

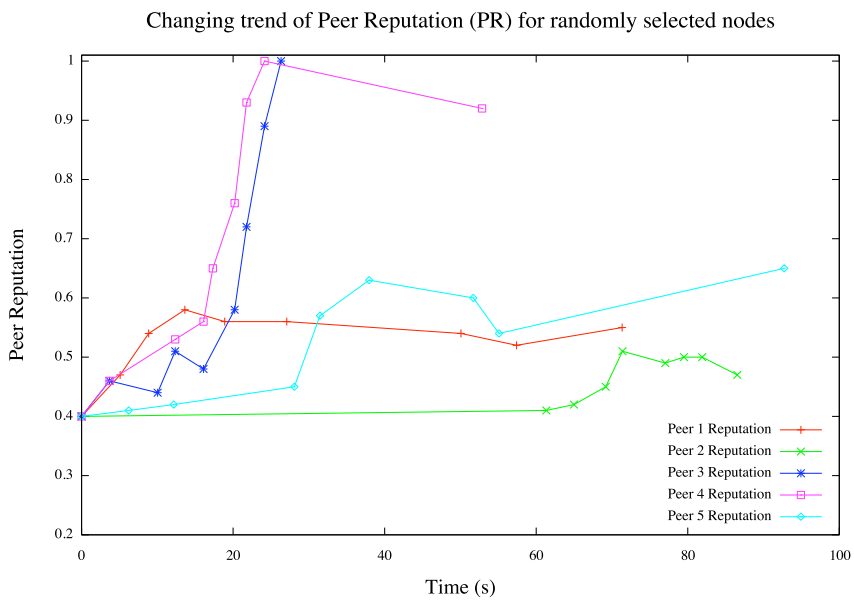

Fig. 5. Peer Reputation for five sample nodes selected randomly to illustrate the changing trends. In this particular experiment we set a minimum QoC of $40 \%$. Therefore, messages with a QoC below this threshold are filtered. As a result, the Peer Reputation cannot go below $40 \%$ for any node.

updated. This value varies between 0 and 1 . Moreover, in order to take into account the active participation of a node in terms of the number of messages sent, we define Participation Factor $p f$ as shown in Equation 9. In this formula $i$ is number of messages received from a particular node for the total simulation time. We use a logarithmic function to map $i$ to a value between 0 and 1 . Moreover, the logarithmic function makes a gradual increase from 0 to 1 . The Participation Factor is directly proportional to the change in PR. In Equation 9, $T$ is the Reputation Threshold and $i$ corresponds to the number of messages received from a specific node. Messages with a QoC greater than $T$ increase PR and vice versa. Figure 5 illustrates the changing trends of PR for randomly selected nodes in our simulation.

$$
\begin{gathered}
p f=1-\frac{\log _{2}(i)+1}{i} \\
P R(n)_{i}=P R(n)_{i-1}+ \\
(Q o C(m)-T) \times \frac{P R(n)_{i-1} \times p f}{\text { HopCount }}
\end{gathered}
$$

\section{Aggregated Quality $(A Q)$}

Aggregated Quality is the combination of the quality of a message which is being transmitted in a network and the reputation of the sender. We can assign variable weights $\omega$ for each of the factors according to the requirements of an application. For example on a highway, vehicles interact with each other for a longer period of time as compared to the vehicles in a city. Thus, on a highway the weight of PR should be higher than the weight of QoC.

$$
A Q(m)=\frac{\omega_{Q o C} Q o C(m)+\omega_{P R} P R(n)}{\omega_{Q o C}+\omega_{P R}}
$$

As illustrated in Figure 6, in addition to QoC filtering and AQ selection we also perform outlier detection of messages. The QoC bound includes all the messages with the minimum 


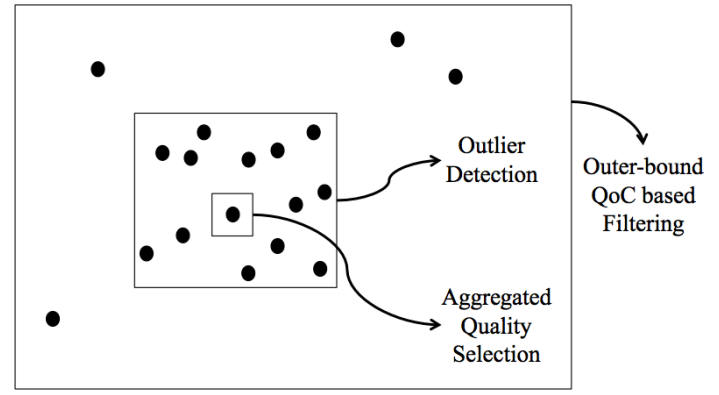

Fig. 6. Outlier detection based on QoC filtering and AQ selection. A dot in this figure represents a location-based message with a certain quality of context produced by a node with a certain reputation. The outer bound shows filtering of messages based on QoC. The messages outside this bound do not meet the minimum required quality. The middle bound shows the outlier detection and the inner bound shows selection of a messages based on AQ. AQ selection keeps the message with the highest AQ value and discards the rest.

level of quality as per the demands of the application. The $\mathrm{AQ}$ bound includes the message with highest $\mathrm{AQ}$ value which in fact is a weighted combination of QoC and PR. As a result we filter out a considerable amount of messages with limited quality. However, there is a chance of losing the best information due to the low reputation of the information provider.

\section{Simulated Evaluation}

In order to evaluate our scheme and to observe the effects of exploiting Quality of Context and Peer Reputation, we simulated a network of vehicles that can communicate wireless and spread information between each other. In our simulation, there are several vehicles that move on the roads and make contact with other vehicles in their vicinity. On the one hand, these vehicle share useful information such as information about parking spaces, traffic jams, accidents, etc. with each other in order to help the vehicles in decision making and choosing their route. On the other hand, we calculate the Quality of Context for each message received at each node in the network and subsequently, we update the Peer Reputation for the sender of that message. Based on the calculated quality values for the message's context information and for the sending node, a vehicle can decide whether to receive, forward or drop the message.

We use a combination of normal and group-based communication in a network of vehicles for our simulated experiments. In the group-based communication, we form groups of vehicles based on their context information such as location and direction, and also based on their interests in information. Each group is represented by vehicles with special responsibilities regarding inter-group and intra-group communication. In addition to group-based communication, we further optimize information dissemination using our intelligent algorithm (Relevance Backpropagation) for adaptive context-aware communication. It is a feedback-based algorithm for identifying and sharing useful information among mobile nodes. Further information about our relevance backpropagation can be found in [9], [10].
In this section, first we describe our experimental setup and then we explain and analyze the results of the simulations.

\section{A. Experimental Setup}

We set up a simulation environment to evaluate our scheme in using Quality of Context and Peer Reputation in a large scale vehicular network. We simulated a network of vehicles that can communicate wirelessly using a Dedicated Short Range Communication (DSRC) wireless network. This is commonly used for vehicle-to-vehicle and vehicle-toinfrastructure communication. It operates over $5.9 \mathrm{GHz}$ band having a transmission range from $10 \mathrm{~m}-1000 \mathrm{~m}$ and a transmission rate of $25 \mathrm{Mbps}-0.25 \mathrm{Mbps}$. It is tailored for high-speed mobile environments.

The dataset used for evaluation is a set of traces from the multi-agent microscopic traffic simulator(MMTS) ${ }^{1}$ developed by $\mathrm{K}$. Nagel. It is capable of simulating traffic over real regional road maps of Switzerland with a high level of realism. The behavior (movements) of the vehicles is reproduced for a period of 24 hours. Individuals in the simulation are distributed over cities and villages in an area of $250 \mathrm{~km} \times 260 \mathrm{~km}$. All individuals choose a time to travel and a route according to where they live and current road congestion. The complete dataset contains 260,000 vehicles with in total more than $25,000,000$ recorded direction and speed changes.

We simulated a subset of 200 vehicles in our experiments which move in a geographical area of $20 \mathrm{~km} \times 10 \mathrm{~km}$ with a high level of realism. All of them send and receive messages. There are three different applications involved with different information interests. The information interests include: (i) parking information, (ii) accident information and (iii) traffic information. We also simulated sparse(off-peak) and dense(peak) traffic situations in order to see the effects of the total number of vehicles on our system.

We performed our simulations using OMNeT++ [8], a component-based, open-architecture discrete event network simulator written in $\mathrm{C}++$. OMNeT++ is widely used in academia for the simulation of computer networks. We also used MiXiM to simulate wireless communication in a vehicular network. MiXiM is an OMNeT++ modeling framework which helps to simulate mobile wireless networks.

\section{B. Results and Analysis}

We have two types of simulated experimental results where the first one is about network traffic usage and the second one shows the number of dropped messages using our two-fold approach. In both simulated experiments we use five different quality settings and assigned equal weights for all the quality factors discussed earlier. These weights might change during our simulated experiments based on a situation. For example, a higher value of weight was assigned to the priority of information factor if in case an emergency traffic message had to be disseminated in the network.

\footnotetext{
${ }^{1}$ http://www.lst.inf.ethz.ch/research/ad-hoc/car-traces/
} 


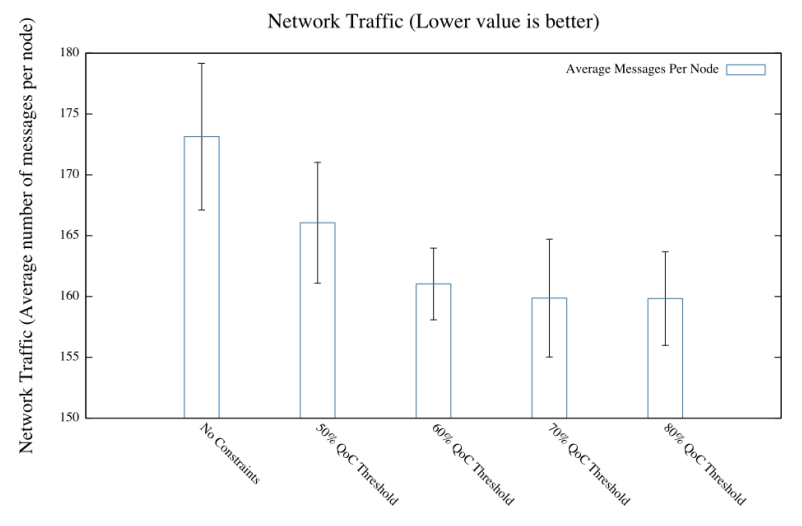

Experimental setups for filtering based on Aggregated Quality

Fig. 7. Average Network Traffic usage per node. This graph depicts average network traffic usage for different filtering thresholds based on Aggregated Quality which is a combination of Quality of Context and Peer Reputation. The first bar is the setup without any filtering based on Aggregated Quality. The results are shown with $95 \%$ confidence interval.

The first setup is without using any quality constraints to filter-out messages. The remaining four setup use different QoC thresholds from $50 \%$ up to $80 \%$. The lowest threshold value is for applications in which QoC is not critical but important, such as traffic flow control on highways. The highest threshold value is chosen for QoC critical applications such as emergency team deployment.

In order to perform the statistical analysis we ran 3 iterations for each of our simulated experiments with random seed value. The analysis shows the averages of our results with a 95\% confidence interval as illustrated in Figure 7 and Figure 9.

We use our relevance backpropagation algorithm for intelligent processing, filtering and routing of messages in our simulation. This way we already discard the redundant and irrelevant information from the network influencing the outcome for network traffic utilization using our two-fold solution. Therefore, we in this paper will only focus on the outcome of our two-fold solution based on the quality of the information and the reputation of the nodes involved in the communication.

In Figure 7, we can see a reduction of approximately $5 \%$ in network traffic utilization for $50 \%$ QoC threshold in comparison to no quality constraints in place. We can further decrease the network traffic usage up to $8 \%$ by increasing the QoC threshold to $80 \%$. This means that nodes in our simulation will produce significantly less information with our quality constraints in place on top of our relevance backpropagation algorithm without effecting the delivery ratio. There is no significant difference in the outcome between the QoC threshold 70\% and $80 \%$ because we have a very few messages in our simulation with $\mathrm{QoC}$ value more than $70 \%$.

As Figure 8 depicts, the number of dropped messages as a result of QoC filtering is directly proportional to QoC threshold. This means that number of dropped messages will increase by increasing the minimum acceptable QoC

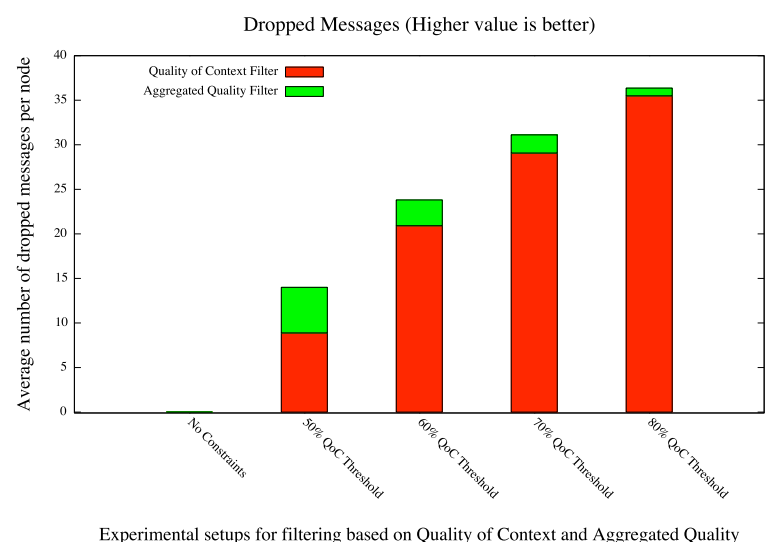

Fig. 8. Average number of dropped messages per node. This graph depicts the average number of dropped messages for different filtering thresholds based on Quality of Context and Aggregated Quality which is a combination of Quality of Context and Peer Reputation. The first bar is for the setup without any quality constraints. The results are shown with $95 \%$ confidence interval.

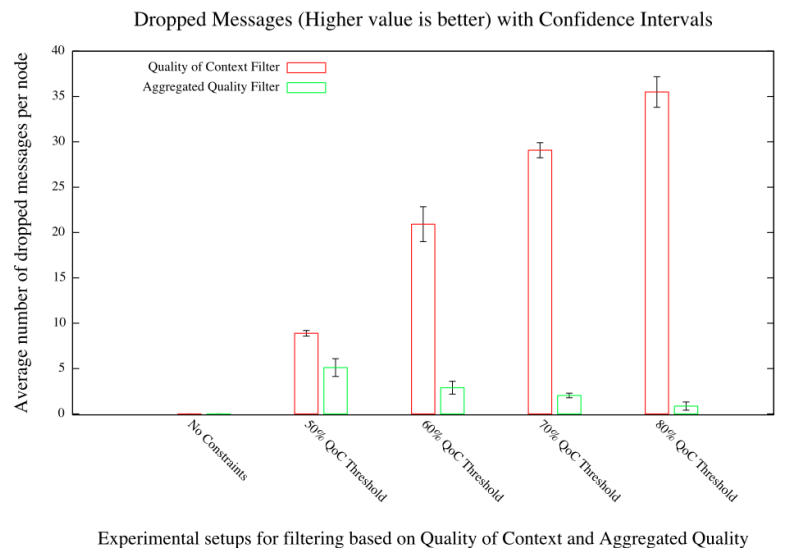

Fig. 9. Average number of dropped messages per node with $95 \%$ confidence interval. This graph depicts the average number of dropped messages for two different filters based on Quality of Context and Aggregated Quality with error bars.

threshold. However, the number of message dropped due to AQ filtering is inversely proportional to QoC threshold. The reason is that more messages are already dropped by $\mathrm{QoC}$ filtering. Therefore, AQ filter has fewer messages with higher QoC and drops fewer message. This concept is illustrated earlier in Figure 6, the messages outside the QoC bound represent the red bars in Figure 8. Similarly, the messages inside QoC bound and outside the AQ bound represent the green bars.

\section{RELATED WORK}

In order to ensure the relevancy of information several QoC requirements have been identified along with context models to tailor these requirements[6], [1], [3], [5]. One of the un-tapped issues in these research works is lack of an evaluation mechanism for the quantification of the QoC requirements. Sheikh et al. [7], identify several quality indicators like precision, freshness, spatial resolution, temporal 
resolution and probability of correctness. The authors proposed that these quality indicators are well-suited in health related pervasive systems. But no quantification mechanism has been proposed by the authors in order to evaluate these parameters. Whereas, in our mechanism we provide several quality requirements for large scale vehicular networks. In our work we also present a detailed evaluation mechanism to be able to validate our quality requirements.

Kim et al. [2], present the quality dimensions such as accuracy, completeness, representation consistency, access security and up-to-dateness for measuring Quality of Context in ubiquitous environments. The authors described a statistical estimation method to compute the accuracy of the data provided by the sensors in a smart home. The evaluation mechanism provided in this work is only suitable under circumstances where a continuous data is being generated by the sensors. Similarly completeness measure also revolves around the ratio of available attributed provided by the sensors by the total number of attributes. Thus, in general the mechanism proposed by the authors is not valid for dynamic, large scale and ephemeral networks. Whereas, our approach is applicable to such networks. We compute the Aggregated Quality by into account both the Quality of Context and the Peer Reputation involved. Thus, in our mechanism we have an additional filtering step which ensures the quality of information provided and the reputation of the source.

Buchholz et al. [1], present a set of generic Quality of Context parameters in close relation to the quality of service and the quality of device without any evaluation framework. The authors later discuss the interdependence between these concepts which are unequal but not unrelated. The authors also strongly emphasis that these concepts help define the QoC agreements which are important for the smooth provisioning of context-aware services in pervasive environments. Our approach focuses on evaluating the Quality of Context and the Peer Reputation for highly dynamic and large scale ubiquitous systems.

\section{CONCLUSION AND Future WORK}

Communication in VANETs faces certain challenges such as having unknown sources of information, having imprecise, erroneous and ambiguous information, massive amount of information flow and an intensive decision making process. In this paper we defined Quality of Context (QoC) and Peer Reputation (PR) for nodes in vehicular networks and propose ways to apply them for efficient communication. We provide a unique two-fold solution in which on the one hand we focus on the quality of the information and on the other hand we aim at determining the reputation of the nodes involved in the communication. The main contributions of our work are to define bounds of acceptable information in order to guarantee a minimum quality of information and eliminate erroneous, ambiguous and imprecise information provided by unknown entities.

Based on our simulated experiments, in Section IV, we can summarize that by using our solution, we firstly reduce the network traffic usage by filtering-out the messages with limited quality and secondly ease out the decision making process for the nodes in VANETs by providing them with less inconsistent information.

As a part of future work we intend to extend our mechanism with more application specific quality constraints. We also plan to further investigate our approach in other types of networks such as MANETs.

\section{ACKNOWLEDGEMENTS}

This research is partially funded by the Interuniversity Attraction Poles Programme Belgian State, Belgian Science Policy, and by the Research Fund K.U.Leuven.

\section{REFERENCES}

[1] T. Buchholz and M. Schiffers, "Quality of context: What it is and why we need it," in In Proceedings of the 10th Workshop of the OpenView University Association: OVUA03, 2003.

[2] Y. Kim and K. Lee, "A quality measurement method of context information in ubiquitous environments," in Proceedings of the 2006 International Conference on Hybrid Information Technology - Volume 02 , ser. ICHIT '06. Washington, DC, USA: IEEE Computer Society, 2006, pp. 576-581.

[3] M. Krause and I. Hochstatter, "Challenges in modelling and using quality of context (qoc)," in MATA, ser. Lecture Notes in Computer Science, T. Magedanz, A. Karmouch, S. Pierre, and I. S. Venieris, Eds., vol. 3744. Springer, 2005, pp. 324-333.

[4] F. Li and Y. Wang, "Routing in vehicular ad hoc networks: A survey," Vehicular Technology Magazine, IEEE, vol. 2, no. 2, pp. 12 -22, 2007.

[5] S. McKeever, J. Ye, L. Coyle, and S. Dobson, "A context quality model to support transparent reasoning with uncertain context," in Proceedings of the 1st international conference on Quality of context ser. QuaCon'09. Berlin, Heidelberg: Springer-Verlag, 2009, pp. 6575.

[6] R. Neisse, M. Wegdam, and M. v. Sinderen, "Trustworthiness and quality of context information," in Proceedings of the 2008 The 9th International Conference for Young Computer Scientists. Washington, DC, USA: IEEE Computer Society, 2008, pp. 1925-1931.

[7] K. Sheikh, M. Wegdam, and M. V. Sinderen, "Quality-of-context and its use for protecting privacy in context aware systems," Journal of Software, vol. 3, 2008.

[8] A. Varga et al., "The OMNeT++ discrete event simulation system," in Proceedings of the European Simulation Multiconference (ESM2001), 2001, pp. 319-324.

[9] A.-U.-H. Yasar, D. Preuveneers, and Y. Berbers, "Evaluation framework for adaptive context-aware routing in large scale mobile peer-topeer systems," Peer-to-Peer Networking and Applications, pp. 1-13, 2010, 10.1007/s12083-010-0090-2.

[10] A.-U.-H. Yasar, Y. Vanrompay, D. Preuveneers, and Y. Berbers, "Optimizing information dissemination in large scale mobile peer-topeer networks using context-based grouping," in 13th International IEEE Conference on Intelligent Transportation Systems, no. 13. IEEE, September 2010, pp. 1065-1071. 